\title{
Reversing an extracellular electron transfer pathway for electrode-driven acetoin reduction
}

Nicholas M. Tefft ${ }^{1}$ and Michaela A. TerAvest ${ }^{1 *}$

${ }^{1}$ Department of Biochemistry and Molecular Biology, Michigan State University, East Lansing, MI, USA *corresponding author

Address: 603 Wilson Rd., East Lansing, MI, 48823 Email: teraves2@msu.edu

\section{Supporting information}

A map of the plasmid created in this study (Figure S1), an image of a Western blot to detect proteorhodopsin (PR) and butanediol dehydrogenase (Bdh) expression (Figure S2), 2,3butanediol production by aerobically grown Shewanella oneidensis with pBBR-Bdh-PR (Figure S3), a photograph of the bioelectrochemical system (Figure S4), cell density in bioelectrochemical systems over time (Figure S5), and total protein recovery from bioelectrochemical systems at the end of experiments (Figure S6). 


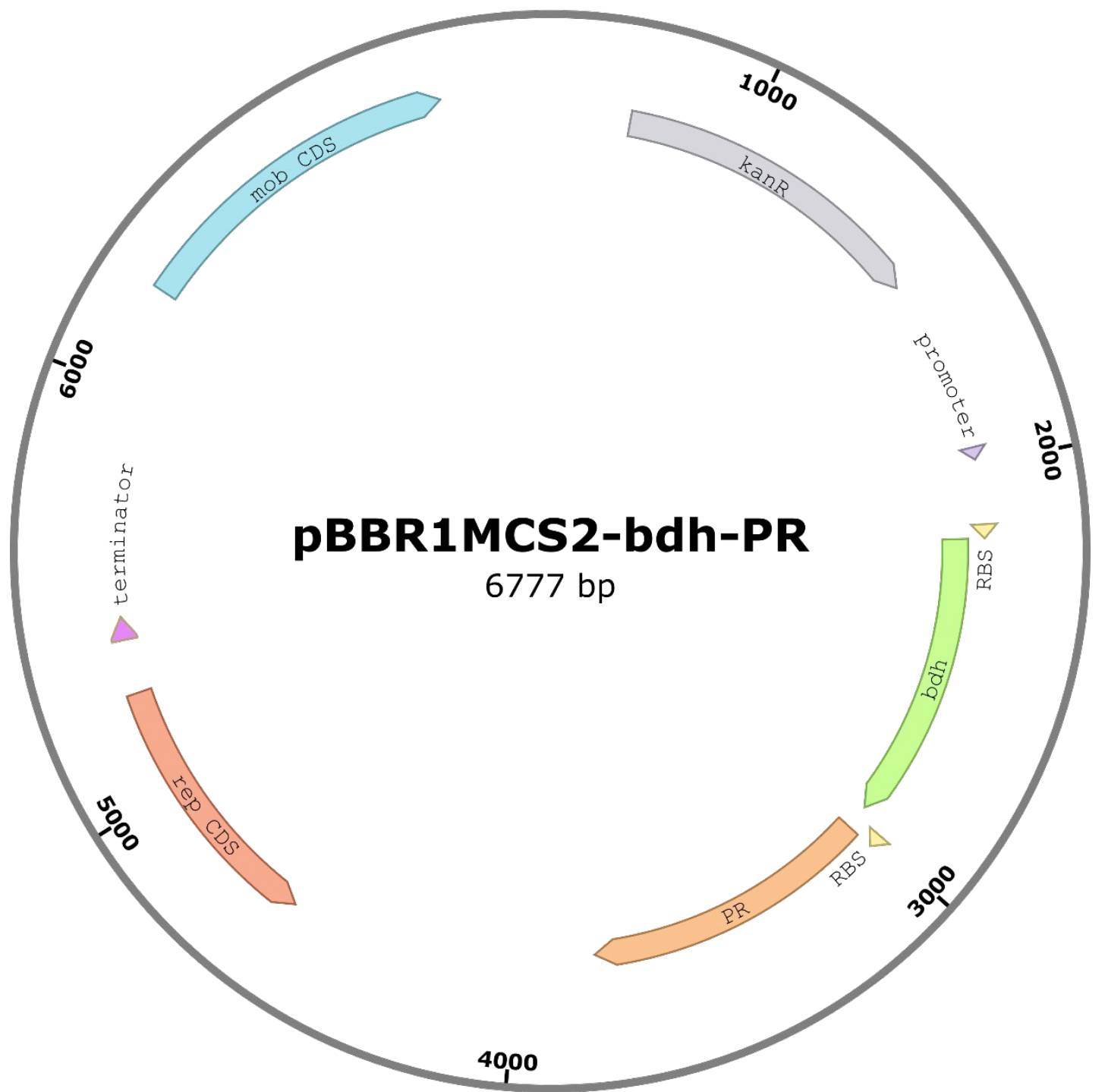

Figure S1. pBBR1MCS2 BDH-PR vector map. $b d h$ and $P R$ inserts were synthesized with optimized RBS sequences for use in Shewanella oneidensis MR-1. Transcription of both genes was controlled by the same constitutive lac promoter. 




\section{BDHBDH}

PR

Figure S2. Expression of Bdh and PR proteins. FLAG tags were added to both genes during synthesis and used in detection of expression by Western blot using anti-FLAG antibodies. 


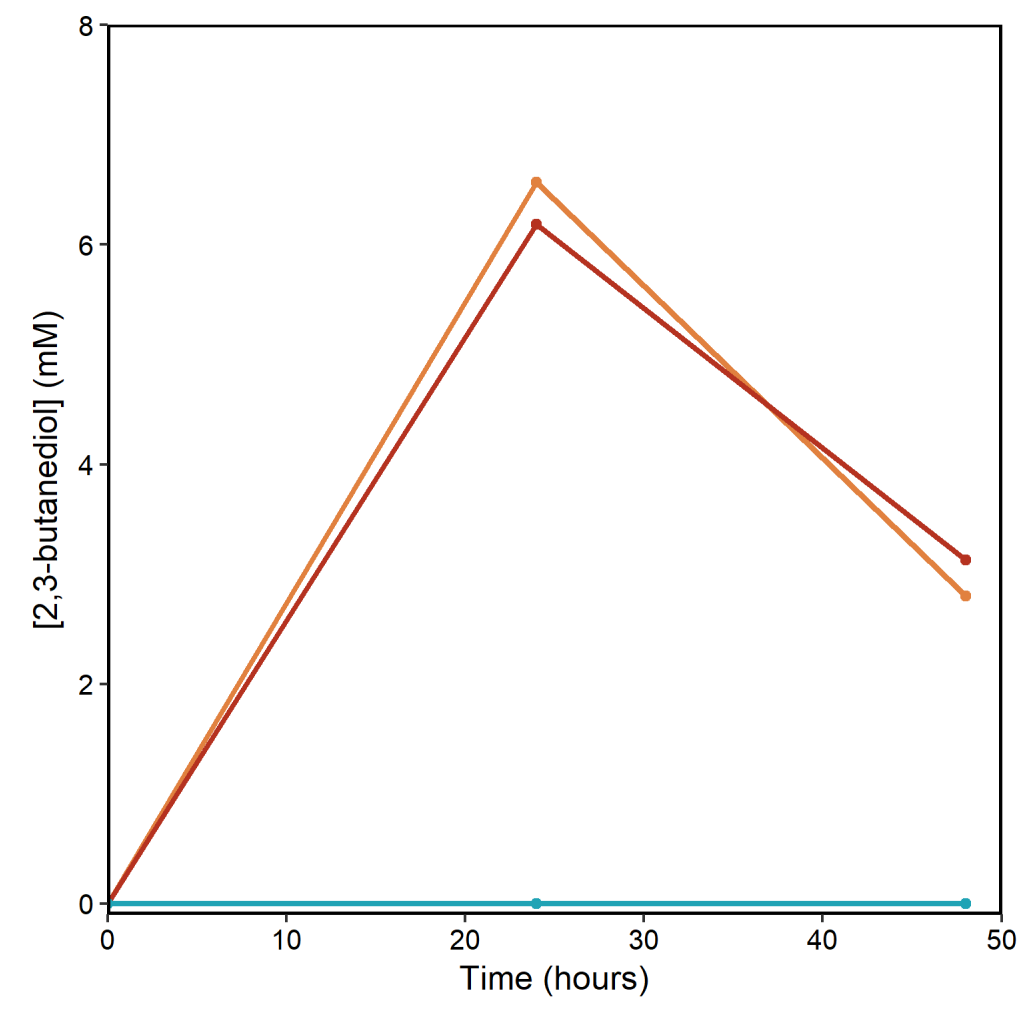

Figure S3. 2,3-butanediol is produced aerobically by cells expressing heterologous Bdh. Modified strains were grown in $50 \mathrm{~mL}$ LB with $15 \mathrm{mM}$ acetoin in 250-mL flasks with $275 \mathrm{rpm}$ shaking. Cells expressing Bdh alone are shown in red. Cells expressing both Bdh and PR are shown in orange, and cells with the empty vector are shown in teal. No detectable 2,3-butanediol was produced by cells lacking Bdh. Results are from single cultures of each strain. 


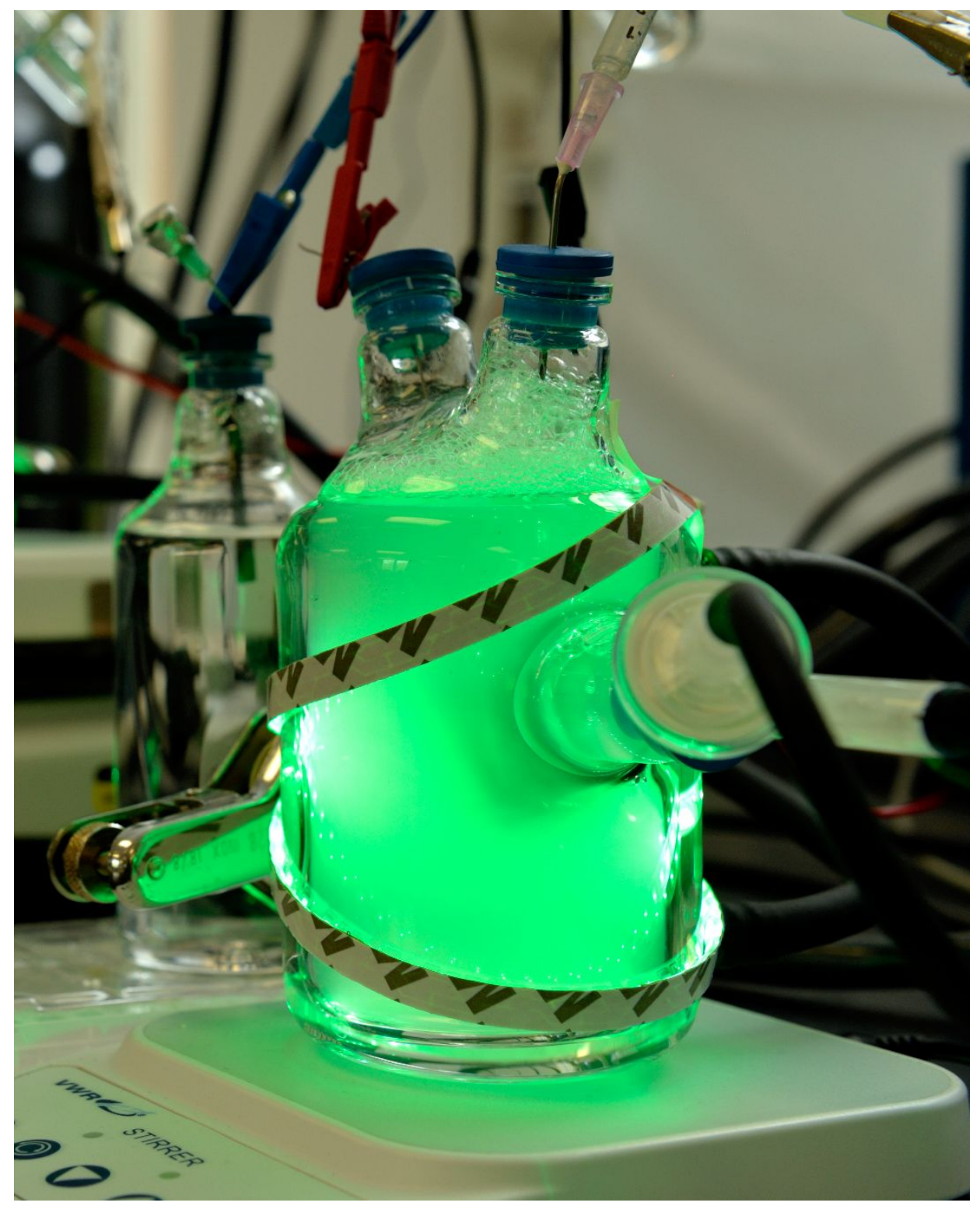

Figure S4. Light is delivered to the system through the outer jacket of the working electrode chamber. Green LED light strips were fixed to the outside of the bioelectrochemical system. Above is a representative picture of this system. 


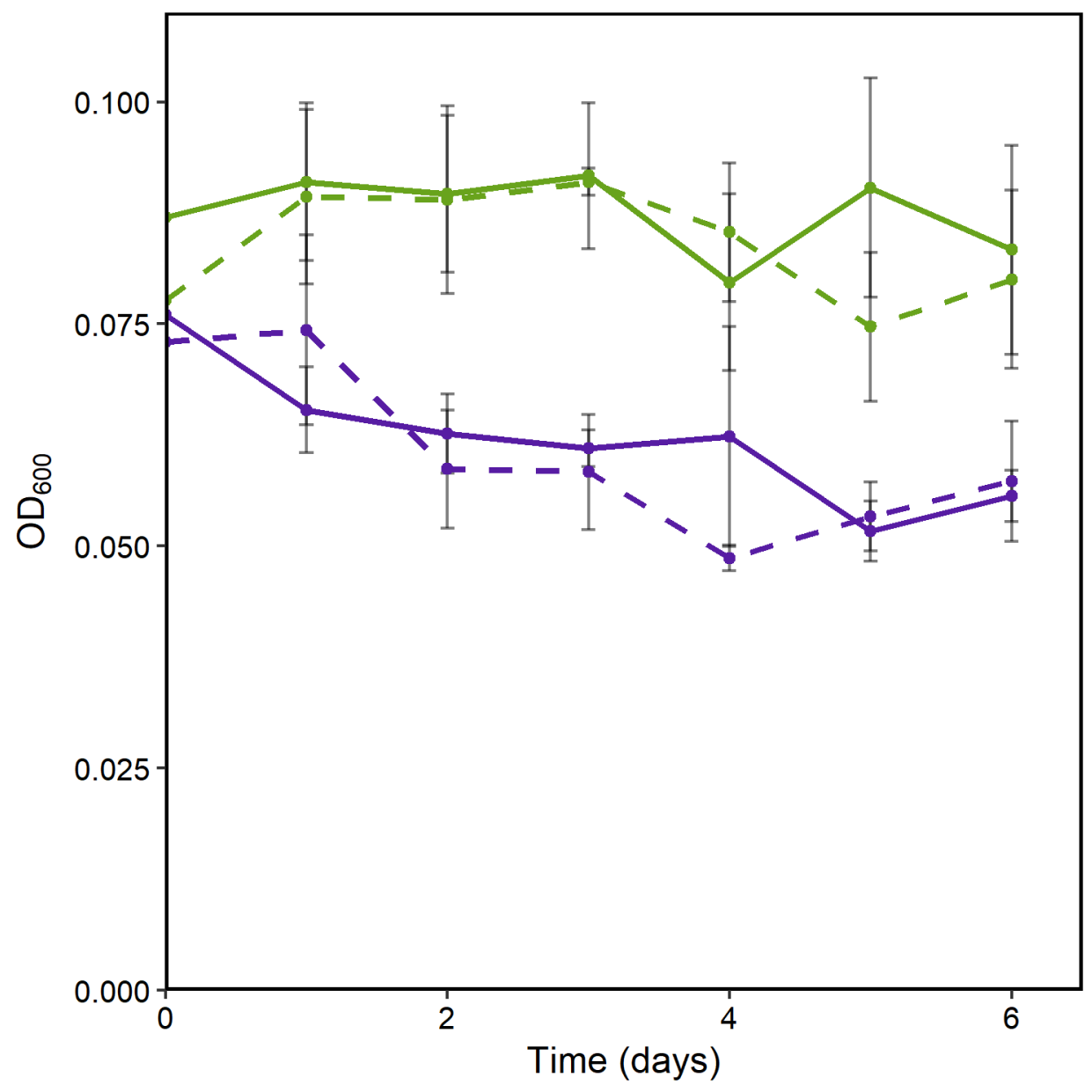

Figure S5. Cell density in bioelectrochemical systems. Samples taken from working electrode chambers with electrodes poised at $-0.3 \mathrm{~V}_{\mathrm{SHE}}$ are shown in solid green and purple lines. Samples taken from bioelectrochemical systems disconnected from the potentiostat are shown in dashed green and purple lines. Samples from reactors containing cells with holo-PR are shown in green and those containing apo-PR are shown in purple. Each point represents the average of three replicates with standard error shown in error bars. 


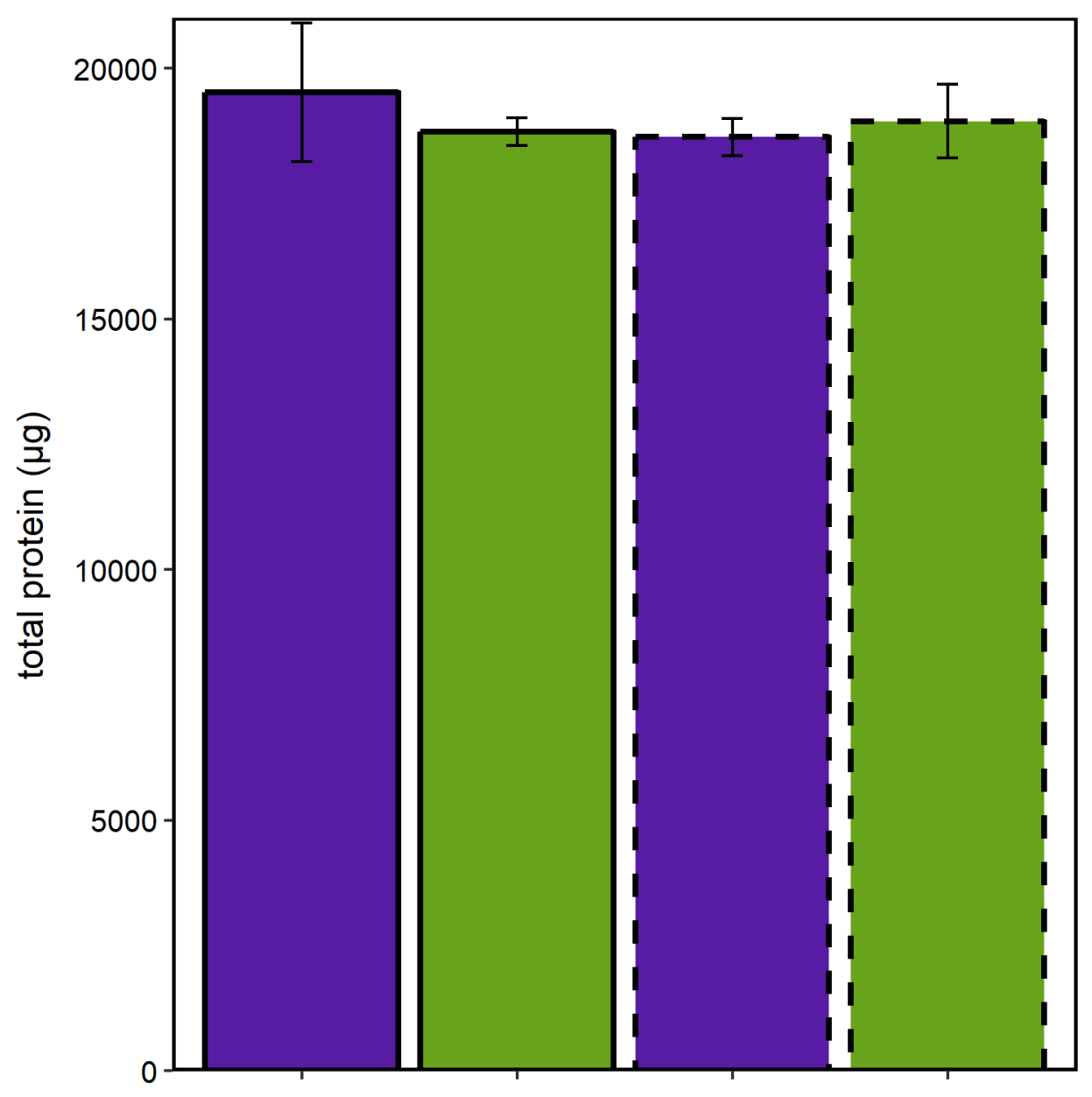

Figure S6. Total protein in bioelectrochemical experiments after 6 days. Total protein measured from samples obtained bioelectrochemical systems after 144 hours. Total protein taken from working electrode chambers with electrodes poised at $-0.3 \mathrm{~V}_{\mathrm{SHE}}$ are shown in solid green and purple bars. Samples taken from bioelectrochemical systems disconnected from the potentiostat are shown in dashed green and purple bars. Samples from reactors containing cells with holo-PR are shown in green and those containing apo-PR are shown in purple. Each bar represents the average of three replicates with standard error shown in error bars. 